\title{
Myocardial Perfusion Reserve in Ischemic Heart Disease
}

B ecause of the aging of the population, cardiovascular disease is still a major cause of death in developed countries and a major cause of morbidity and decreased quality of life despite improved outcome for acute events (1). With the diffusion of new risk factors (physical inactivity, diabetes mellitus, and obesity), the prevalence of coronary artery disease (CAD), the most

\section{See page 214}

frequent among cardiovascular diseases, is actually increasing together with its most relevant consequence, that is, progressive myocardial dysfunction and heart failure. Accordingly, better prevention and management of CAD is needed to further reduce early cardiovascular mortality and morbidity, to prevent development of heart failure, and hence to improve life expectancy and quality of life and reduce health costs. More efficient control of $\mathrm{CAD}$ implies early detection and identification of new, reliable endpoints for prevention and treatment.

\section{CAD AND IHD: CHANGING PARADIGMS}

In everyday clinical practice, the main clinical manifestations of ische-

Received Aug. 26, 2008; revision accepted Sep. 2, 2008.

For correspondence or reprints contact: Danilo Neglia, Clinical PET Laboratory, Fondazione C.N.R. Regione Toscana, G. Monasterio Institute of Clinical Physiology, C.N.R. - National Research

Council, Via G. Moruzzi 1, Pisa, Italy.

E-mail: dneglia@ifc.cnr.it

COPYRIGHT (C) 2009 by the Society of Nuclear

Medicine, Inc.

DOI: 10.2967/jnumed.108.056317 mic heart disease (IHD) (angina, myocardial infarction, cardiac sudden death, and heart failure) are attributed to the effects of epicardial coronary obstructive lesions. In this view, stenotic or occluded coronary arteries hamper downstream blood flow, reduce myocardial perfusion, and cause contractile dysfunction. Accordingly, in patients with ischemic symptoms or heart failure, the primary clinical goal is to assess the presence of significant CAD and to revascularize stenotic arteries.

However, this classic paradigm of equivalence between IHD and CAD is challenged by several facts. There is evidence that the presence and extent of CAD are not always related to the clinical manifestations of IHD, nor are they able to predict its progression and response to treatment (2). On the other hand, endothelial dysfunction can result in coronary vascular and blood flow abnormalities not necessarily associated with significant coronary stenosis but still able to cause IHD (3). Coronary abnormalities may be mainly functional or may consist of minor lesions in the vascular wall, detectable only on the direct analysis of vessel structure. Nevertheless, they can reduce coronary vasodilating properties at the macroand microvascular levels, hampering myocardial blood flow (MBF). Coronary functional and microvascular alterations may coexist with epicardial coronary artery lesions and contribute to ischemia (3).

\section{WHY MEASURE MPR IN PATIENTS WITH SUSPECTED IHD?}

Despite the pathogenetic complexity of myocardial ischemia, the diagnostic work-up of patients with suspected IHD is still oriented mainly toward the detection of anatomic CAD. This anatomically oriented approach may negatively affect patient management, leading to suboptimal treatment, additional risks, and increased health costs. Recent results in patients with IHD confirm that, in the presence of documented $\mathrm{CAD}$, revascularization improves the survival rate over optimal medical treatment only in patients with moderate to severe ischemia as evidenced by perfusion imaging $(4,5)$.

According to these concepts, in patients with suspected IHD a new strategy that takes advantage of the evolving imaging technology is warranted-a strategy that has the goal of identifying reliable markers of disease progression and new endpoints for treatment. Early or minimal coronary atherosclerosis can be manifested by diffuse abnormalities of endothelial or microvascular function leading to impairment of myocardial perfusion. At these stages, quantitative PET, which is able to show global impairment of absolute MBF and myocardial perfusion reserve (MPR), can be used to stratify prognosis (6) and to indicate and monitor aggressive medical treatment. When atherosclerosis progresses, the arterial wall becomes abnormal and the coronary lumen narrows, and these changes can be noninvasively detected by multislice CT coronary angiography (MSCT). The combination of MSCT with perfusion imaging, either with SPECT or PET, may identify coronary stenoses that limit blood flow during stress and hence require revascularization (7). This combined approach is also able to document impairment of myocardial perfusion not directly related to obstructive coronary lesions but that nevertheless requires aggressive medical treatment. 


\section{MPR AND PROGNOSIS}

In this issue of The Journal of Nuclear Medicine, Tio et al. (8) assessed the prognostic impact of PETmeasured MPR in patients with IHD. In a large, retrospective population of patients with known CAD who underwent a flow-metabolism PET study, after which they were not candidates for coronary revascularization, the authors documented a significantly worse prognosis in those subjects with a higher reduction in global MPR. After adjustment for age and sex, MPR was associated with a hazard ratio for cardiac death of 4.11 whereas the risk for left ventricular ejection fraction was 2.76 . MPR showed independent prognostic power also when the composite endpoint of major adverse cardiovascular events (including cardiac death, myocardial infarction, percutaneous coronary intervention, coronary artery bypass grafting, or hospitalization for acute coronary syndrome or heart failure) was considered. The authors concluded that patients with IHD with a low MPR are at high risk of cardiac death and that in this population MPR is a more sensitive predictor than left ventricular ejection fraction.

The study population was characterized by a high prevalence of old myocardial infarction $(71 \%)$ and moderate to severe heart failure (New York Heart Association class II, 49\%; class III-IV, $36 \%$ ) with depressed left ventricular systolic function (left ventricular ejection fraction, $36 \% \pm 15 \%)$. In these kinds of patients, it is commonly accepted that prognosis depends mainly on the extent of regional viability and ischemia downstream from a coronary lesion. Accordingly, revascularization is indicated in the presence of a sufficient amount of viable and ischemic myocardium subtended by significant coronary stenosis. Actually, the study patients were not revascularized, presumably because of lack of the above criteria. The interesting result is that, in this study cohort, prognosis was indeed dependent on global impairment of myocardial perfusion and independent of the extent of regional ischemia, viability, and left ventricular dysfunction.

These results (8) add an important piece of evidence to the concept that global impairment of myocardial perfusion is a common finding in patients with left ventricular dysfunction due either to "primitive" myocardial disease (9) or to CAD (10) and, more important, that global reduction of coronary vasodilating capability is a powerful independent determinant of prognosis $(11,12)$. As Tio et al. underline, one could question what the clinical significance of MPR measurements could be in patients with CAD that had been judged not amenable to coronary intervention. It can be argued that low MPR may push one to reconsider a coronary intervention and to optimally treat patients with an aggressive approach aimed at improving endothelial and vascular function. Even if recent results support the crucial importance of optimal medical treatment in CAD (13), further studies are needed to better understand which patient will benefit from complete revascularization or from aggressive medical treatment.

\section{PET-MEASURED MPR: A NEW TARGET OF TREATMENT}

PET has been shown to allow noninvasive and accurate quantification of MBF if suitable tracers are used and appropriate mathematic models applied (14). MPR, the ratio of MBF during near-maximal coronary vasodilation to basal MBF, is an integrated measure of flow through both the large epicardial coronary arteries and the microcirculation. Thus, an abnormal MPR, not regionally related to angiographically demonstrable coronary stenosis, may reflect dysfunction of the coronary microcirculation after exclusion of hemodynamic and extravascular factors. Coronary microvascular dysfunction (15) can be caused by structural or functional changes that may involve the endothelium. Recent advances have highlighted the crucial role of the microcirculation in the pathogenesis of IHD and of other relevant cardiovascular diseases (15). MBF and MPR are globally reduced also in the remote myocardium supplied by angiographically normal coronary arteries in patients with significant CAD elsewhere (10). However, Fujiwara et al. found preserved MPR in regions supplied by angiographically normal coronary arteries in patients with 1-vessel CAD without coronary risk factors (16). It is possible that angiographically undetectable atherosclerosis and endothelial dysfunction, associated with the presence and severity of coronary risk factors, could be responsible for a blunted MPR in patients with or without evident CAD (17). Indeed, it has recently been shown that in patients without clinically detected CAD and with a low to intermediate Framingham risk score, which integrates all major risk factors, MPR assessed noninvasively by quantitative PET is inversely related to estimated 10-y CHD risk (18). In other words, MPR provides a way to document how risk factors translate into measurable damage to the coronary circulation, predicting future cardiovascular events independently of "significant" coronary stenoses.

The pathogenetic relationship between depressed myocardial perfusion and progressive myocardial dysfunction is another field of major recent interest. The classic concept of hibernating myocardium, that is, regional myocardial dysfunction caused by a stenotic coronary artery that limits resting downstream flow and improves on coronary revascularization, is being revisited (19). In some recent studies, a severely impaired MPR that was also evident in hibernating myocardium improved after revascularization in parallel with contractile function. Thus, repetitive ischemia due to reduced MPR may lead to prolonged stunning initially contributing to chronic dysfunction. This hypothesis holds true also in patients with idiopathic left ventricular dysfunction, where ischemia can be attributed essentially to coronary microvascular or endothelial impairment (20). In these patients, reduced MBF and MPR are unrelated to the initial extent of left ventricular dysfunction 
(9) and are independent predictors of subsequent cardiac events associated with an increased relative risk of death, as in the study of Tio et al. (8), and progression of heart failure (11). The microcirculatory ischemia hypothesis is also supported by the recent finding of a flow-metabolism mismatch, that is, reduced myocardial perfusion and enhanced glucose metabolism, invasively assessed by the study of intermediate myocardial metabolism in patients with idiopathic left ventricular dysfunction (21). This pattern is considered a distinctive marker of hibernation in patients with $\mathrm{CAD}$ and heart failure. These similarities between diseases classically designated as myocardial or coronary strongly support a common pathogenetic background characterized by a direct relationship between diffuse impairment of myocardial perfusion at the microvascular level and progressive myocardial dysfunction. This fascinating hypothesis, however, awaits direct confirmative studies.

PET is a unique noninvasive tool to identify abnormal MBF and MPR as new targets of therapy aimed at improving global vascular function. Improvement in myocardial perfusion abnormalities by PET has been documented after long-term intense risk factor modification $(22,23)$ or $\alpha$ - and $\beta$-blockade in patients with left ventricular dysfunction (24).

Further technologic developments may offer more specific targets of treatment in IHD. The recent availability of hybrid PET/MSCT scanners allows combining the anatomic information provided by MSCT, which includes possible assessment of early abnormalities of the coronary vessel walls, with the complete functional information provided by quantitation of myocardial perfusion by PET. A possible new diagnostic integrated approach to patients with suspected IHD, with or without known CAD, by multimodal PET and MSCT imaging before performing invasive coronary angiography could have many advantages (25). On the one hand it could improve our understanding of IHD, and on the other it could optimize patient management and use of health resources. A possible risk could be an overuse and duplication of costly technologies and increased radiation exposure to the patient. Accordingly, there is an urgent need to evaluate the advantages of this approach for accuracy and cost-effectiveness against reference methods and patient outcome.

\section{Danilo Neglia \\ National Research Council Pisa, Italy \\ Antonio L'Abbate Scuola Superiore Sant'Anna Pisa, Italy}

\section{REFERENCES}

1. Scholte Reimer W, Simoons ML, Boersma W, et al. Euro Heart Survey: Cardiovascular Diseases in Europe. Sophia Antipolis, France: European Society of Cardiology; 2006.

2. Chaitman BR, Ryan TJ, Kronmal RA, Foster ED, Frommer PL, Killip T. Coronary Artery Surgery Study (CASS): comparability of 10 year survival in randomized and randomizable patients. $J$ Am Coll Cardiol. 1990;16:1071-1078.

3. L'Abbate A, Sambuceti G, Neglia D. Myocardial perfusion and coronary microcirculation: from pathophysiology to clinical application. J Nucl Cardiol. 2002;9:328-337.

4. Hachamovitch R, Hayes SW, Friedman JD, Cohen I, Berman DS. Comparison of the short-term survival benefit associated with revascularization compared with medical therapy in patients with no prior coronary artery disease undergoing stress myocardial perfusion single photon emission computed tomography. Circulation. 2003;107:2900-2907.

5. Shaw LJ, Berman DS, Maron DJ, et al., for the COURAGE Investigators. Optimal medical therapy with or without percutaneous coronary intervention to reduce ischemic burden: results from the Clinical Outcomes Utilizing Revascularization and Aggressive Drug Evaluation (COURAGE) trial nuclear substudy. Circulation. 2008;117:1283-1291.

6. Schindler TH, Nitzsche EU, Schelbert HR, et al. Positron emission tomography-measured abnormal responses of myocardial blood flow to sympathetic stimulation are associated with the risk of developing cardiovascular events. J Am Coll Cardiol. 2005;45:1505-1512.

7. Di Carli MF, Dorbala S, Hachamovitch R. Integrated cardiac PET-CT for the diagnosis and management of CAD. J Nucl Cardiol. 2006;13: 139-144.

8. Tio RA, Dabeshlim A, Siebelink H-MJ, et al. Comparison between the prognostic value of left ventricular function and myocardial perfusion reserve in patients with ischemic heart disease. J Nucl Med. 2009;50:214-219.

9. Neglia D, Parodi O, Gallopin M, et al. Myocardial blood flow response to pacing tachycardia and to dipyridamole infusion in patients with dilated cardiomyopathy without overt heart failure: a quantitative assessment by positron emission tomography. Circulation. 1995;92:796-804.

10. Sambuceti G, Parodi O, Marzullo P, et al. Regional myocardial blood flow in stable angina pectoris associated with isolated significant narrowing of either the left anterior descending or left circumflex coronary artery. Am J Cardiol. 1993;72:990-994.

11. Neglia D, Michelassi C, Trivieri MG, et al. Prognostic role of myocardial blood flow impairment in idiopathic left ventricular dysfunction. Circulation. 2002;105:186-193.

12. Cecchi F, Olivotto I, Gistri R, Lorenzoni R, Chiriatti G, Camici PG. Coronary microvascular dysfunction and prognosis in hypertrophic cardiomyopathy. N Engl J Med. 2003;349:1027-1035.

13. Boden WE, O'Rourke RA, Teo KK, et al., for the COURAGE Trial Research Group. Optimal medical therapy with or without PCI for stable coronary disease. N Engl J Med. 2007;356:1503-1516.

14. Nitzsche EU, Choi Y, Czernin J, Hoh CK, Huang $S$, Schelbert HR. Noninvasive quantification of myocardial blood flow in humans: a direct comparison of the $\left[{ }^{13} \mathrm{~N}\right] \mathrm{ammonia}$ and the $\left[{ }^{15} \mathrm{O}\right]$ water techniques. Circulation. 1996;93:2000-2006.

15. Camici PG, Crea F. Coronary microvascular dysfunction. N Engl J Med. 2007;356:830-840.

16. Fujiwara M, Tamura T, Yoshida K, et al. Coronary flow reserve in angiographically normal coronary arteries with one-vessel coronary artery disease without traditional risk factors. Eur Heart J. 2001; 22:479-487.

17. Zeiher AM, Drexler H, Wollschläger H, Just H. Endothelial dysfunction of the coronary microvasculature is associated with impaired coronary blood flow regulation in patients with early atherosclerosis. Circulation. 1991;84:1984-1992.

18. Dorbala S, Hassan A, Heinonen T, Schelbert HR, Di Carli MF, for the RAMPART Investigators. Coronary vasodilator reserve and Framingham risk scores in subjects at risk for coronary artery disease. $J$ Nucl Cardiol. 2006;13:761-767.

19. Camici PG, Rimoldi OE. Myocardial blood flow in patients with hibernating myocardium. Cardiovasc Res. 2003;57:302-311.

20. van den Heuvel AV, van Veldhuisen DJ, van der Wall EE, et al. Regional myocardial blood flow reserve impairment and metabolic changes suggesting myocardial ischemia in patients with idiopathic dilated cardiomyopathy. J Am Coll Cardiol. 2000; 35:19-28.

21. Neglia D, De Caterina A, Marraccini P, et al. Impaired myocardial metabolic reserve and substrate selection flexibility during stress in patients with idiopathic dilated cardiomyopathy. Am J Physiol Heart Circ Physiol. 2007;293:H3270-H3278.

22. Gould KL, Ornish D, Scherwitz L, et al. Changes in myocardial perfusion abnormalities by positron emission tomography after long-term, intense risk factor modification. JAMA. 1995;274:894-901.

23. Schindler TH, Facta AD, Prior JO, et al. Improvement in coronary vascular dysfunction produced with euglycaemic control in patients with type 2 diabetes. Heart. 2007;93:345-349.

24. Neglia D, De Maria R, Masi S, et al. Effects of long-term treatment with carvedilol on myocardial blood flow in idiopathic dilated cardiomyopathy. Heart. 2007;93:808-813.

25. Di Carli MF, Dorbala S, Meserve J, El Fakhri G, Sitek A, Moore SC. Clinical myocardial perfusion PET/CT. J Nucl Med. 2007;48:783-793. 